\title{
Fine Mapping of the Dominant Potyvirus Resistance Gene Pvr7 Reveals a Relationship with Pvr4 in Capsicum annuum
}

\author{
Jelli Venkatesh, Jeongtak An, Won-Hee Kang, Molly Jahn, and Byoung-Cheorl Kang† ${ }^{\dagger}$
}

First, second, third, and fifth authors: Department of Plant Science and Plant Genomics and Breeding Institute, College of Agriculture and Life Sciences, Seoul National University, Seoul 151-921, Korea; and fourth author: Department of Agronomy, College of Agriculture and Life Sciences, University of Wisconsin-Madison.

Accepted for publication 12 September 2017.

\begin{abstract}
Pepper mottle virus (PepMoV) is the most common potyvirus infection of pepper plants and causes significant yield losses. The $P v r 7$ gene from Capsicum chinense PI159236 and the Pvr4 gene from C. annuum CM334 both have been reported to confer dominant resistance to PepMoV. The $P v r 7$ locus conferring resistance to PepMoV in C. annuum '9093' was previously mapped to chromosome 10 . To develop a high-resolution map of the Pvr7 locus in 9093 , we constructed an intraspecific $\mathrm{F}_{2}$ mapping population consisting of 916 individuals by crossing PepMoV-resistant C. annuиm '9093' and the PepMoV-susceptible C. апnиum 'Jeju'. To delimit the $P v r 7$ target region, single-nucleotide polymorphism (SNP) markers derived from the Pvr4 region were used for genotyping the $\mathrm{F}_{2}$

population. Molecular mapping delimited the $P v r 7$ locus to a physical interval of $258 \mathrm{~kb}$, which was the same region as $P v r 4$ on chromosome 10. Three SNP markers derived from Pvr4 mapping perfectly cosegregated with PepMoV resistance. Sequencing analyses of the $P v r 7$ flanking markers and the $P v r 4$-specific gene indicated that $P v r 7$ and $P v r 4$ are the same gene. Resistance spectrum analysis of 9093 against pepper potyviruses showed that 9093 has a resistance spectrum similar to that of cultivar CM334. These combined results demonstrate that, unlike previously thought, the dominant PepMoV resistance in 9093 could be derived from C. annuum 'CM334', and that Pvr4 and Pvr7 should be considered as the same locus.
\end{abstract}

Potyviruses are the major group of viruses infecting plants, and are transmitted by aphid vectors (Valkonen et al. 1996). Pepper mottle virus (PepMoV), which belongs to the Potyvirus genus, often infects pepper (Abdalla et al. 1991; Dogimont et al. 1996; Lamb et al. 2001; Rialch et al. 2015). Plants infected with PepMoV display a range of symptoms, including mosaic and dark-green vein banding, crinkled leaves, and stunted plant growth (Kim et al. 2010). PepMoV is most prevalent in North America and Asia, and causes serious economic losses in pepper (Kim et al. 2009; Moury et al. 2005; Rodríguez-Alvarado et al. 2002; Warren and Murphy 2003).

Several potyvirus resistance $(R)$ genes have been reported in pepper (Caranta et al. 1996; Grube et al. 2000; Kyle and Palloix 1997; Lee et al. 2013). For instance, recessive potyvirus $R$ genes encoding eukaryotic translation initiation factors have been identified in Capsicum spp. (Hwang et al. 2009; Kang et al. 2005; Murphy and Kyle 1995; Ruffel et al. 2006). The pvrl gene, which was identified in Capsicum chinense PI159236 and PI152225, confers resistance to Tobacco etch virus (TEV) HAT isolate, Potato virus $Y$ (PVY) pathotype 0, and PepMoV (Kyle and Palloix 1997; Murphy et al. 1998; Yeam et al. 2005). The gene $p v r 1^{1}$ was identified in C. annuum 'Yolo Y' and confers resistance to PVY (Kang et al. 2005; Ruffel et al. 2002; Yeam et al. 2005). The pvr3 gene was reported in $C$. annuum 'Avelar' and confers resistance to PepMoV (Guerini and Murphy 1999; Murphy and Kyle 1995; Parrella et al. 2002). Pepper plants expressing the pvr6 gene from C. annuum 'Perennial' are resistant to Chilli veinal mottle virus

${ }^{\dagger}$ Corresponding author: B.-C. Kang: E-mail: bk54@snu.ac.kr

J. Venkatesh and J. An contributed equally to this work.

*The $\boldsymbol{e}$-Xtra logo stands for "electronic extra" and indicates that two supplementary figures are published online.

(c) 2018 The American Phytopathological Society
(Caranta et al. 1996; Hwang et al. 2009; Ruffel et al. 2006). In addition to these recessive sources of resistance, dominant potyvirus $R$ genes also have been reported in pepper. The Pvr4 gene was identified in C. annuum 'CM334' and confers resistance to PepMoV (Caranta et al. 1999; Grube et al. 2000; Kim et al. 2017). Pvr7 was identified as tightly linked to Pvr4 in C. chinense 'PI159236' (Grube et al. 2000).

Candidate genes for Pvr4 have been identified by Kim et al. (2017) using a map-based cloning approach. Transient coexpression analyses of the putative candidate genes in Nicotiana benthamiana showed that a nucleotide-binding leucine-rich repeat (NB-LRR)type disease $R$ gene (CaNBARC322) is responsible for hypersensitiveresponse-mediated resistance (Kim et al. 2017). The Pvr4 gene consists of seven exons encoding a typical coiled-coil NB-LRR family protein, whereas the susceptible allele $p v r 4$ from $C$. annuиm 'ECW' consists of six exons (Kim et al. 2017).

$P v r 4$ and $P v r 7$ were originally reported as tightly linked but nonallelic due to the apparent identification of a small number of putative recombinant plants in a segregating population (Grube et al. 2000). In that study, Pvr4 and Pvr 7 were localized on the lower arm of chromosome 10 , approximately 15 centimorgans (cM) from the random amplified polymorphic DNA marker Q6, which is tightly linked to Tomato spotted wilt virus (TSWV) resistance gene Tsw (Grube et al. 2000; Jahn et al. 2000). Although previous research showed that $P v r 7$ is located on chromosome 10, the precise location of $P v r 7$ in the pepper genome and the gene encoding $P v r 7$ have not previously been identified. The widely used 9093 line carrying $P v r 7$ was derived from repeated backcrossing of a belltype $C$. annuum recurrent parent line to $C$. chinense 'PI159236' (Grube et al. 2000). Genetic fine mapping of the $P v r 7$ locus promises to broaden our molecular and genetic understanding of the dominant inheritance mechanism, and could facilitate the breeding of PepMoV-resistant varieties.

In this study, we performed fine mapping of the $P v r 7$ locus using $9169093 \times \mathrm{Jeju}_{2}$ plants derived from a cross between the PepMoVresistant parental line C. annuum '9093' and the susceptible parental 
line $C$. annuum 'Jeju'. Our fine mapping localized the $P v r 7$ locus to a physical interval of $258 \mathrm{~kb}$ flanked by the single-nucleotide polymorphism (SNP) markers SNP-H2.3 and SNP-H1.7 on chromosome 10, in which Pvr4 also was localized (Kim et al. 2017). To determine the relationship between Pvr4 and Pvr7, we investigated the resistance spectrum of 9093 using other pepper viruses, including TSWV and TEV. The results of viral resistance spectrum analysis, genetic fine mapping, and sequence characterization of the Pvr4 gene-specific insertion and deletion (InDel) and the $P v r 7$ flanking markers all indicate that $P v r 7$ and $P v r 4$ are, in fact, the same gene; hence, we suggest the revision of the $P v r 7$ designation to be Pvr4.

\section{MATERIALS AND METHODS}

Plant materials and construction of mapping population. Five Capsicum accessions were used for viral resistance spectrum and fine-mapping analysis, including $C$. chinense 'PI159236' and 'PI152225' and C. annuum 'ECW', '9093', and 'Jeju'. PI159236 and PI152225 harbor resistance to PepMoV (Grube et al. 2000), whereas ECW is susceptible to PepMoV (Kim et al. 2011). To perform a genetic analysis and fine mapping of the $P v r 7$ gene, $\mathrm{F}_{1}$ and $F_{2}$ populations were developed by crossing the PepMoVresistant $C$. annuum ' 9093 ' to the PepMoV-susceptible $C$. annuum 'Jeju'. In total, $9169093 \times \mathrm{Jeju} \mathrm{F}_{2}$ plants were used to map the $P v r 7$ locus. C. annuum 'CM334' and 'B2110-1A' and commercial $\mathrm{F}_{1}$ hybrid IPH10023 were kindly provided by Bob Heisey (United Genetics Seeds Co., Hollister, CA) and used to validate the identity of the Pvr7 gene.

Inoculum preparation and plant inoculation. PepMoV strain Vb1 (hereafter PepMoV) and TEV were maintained in $N$. benthamiana. TSWV was maintained in N. rustica. Approximately $1 \mathrm{~g}$ of infected leaf tissue was ground on ice in $5 \mathrm{ml}$ of ice-cold $0.1 \mathrm{M}$ potassium phosphate buffer $(\mathrm{pH} 7.0)$ mixed with 400-grit carborundum, manually rubbed on fully expanded leaves of experimental plants, allowed to stand for $20 \mathrm{~min}$, and then washed off with distilled water.

Either the first or second pair of true leaves of 3- to 4-week-old pepper plants were inoculated. Inoculated plants were grown in a growth chamber maintained at 23 and $21^{\circ} \mathrm{C}$ (day and night, respectively) with $16 \mathrm{~h}$ of light provided by cool fluorescent lighting. Inoculated pepper plants were monitored for development of viral symptoms up to 21 days postinoculation (dpi). In total, 10 each of PI159236, PI152225, 9093, Jeju, and $9093 \times$ Jeju $F_{1}$ plants were inoculated with TSWV and TEV to investigate the virus-resistance spectrum of $P v r 7$.

Double-antibody sandwich enzyme-linked immunosorbent assay. Enzyme-linked immunosorbent assay (ELISA) tests were performed at 7, 8, and 13 dpi to detect the viral coat proteins of TSWV, TEV, and PepMoV. ELISA tests were performed according to the manufacturer's instructions (Agdia, Elkhart, IN). Samples were collected from inoculated and noninoculated upper leaves. The ELISA tests were performed three times.

Monitoring viral infection using PepMoV tagged with green fluorescent protein. PepMoV constructs fused to green fluorescent protein (GFP) were used to monitor the systemic spread of PepMoV. GFP was visualized in infected plants with the MultiDocIt imaging System (UVP, LLC, Upland, CA).

Genomic DNA extraction. Total genomic DNA was extracted from fully expanded cotyledon tissue of each plant using the cetyltrimethylammonium bromide method (Kang et al. 2010). DNA concentration and quality were analyzed using the NanoDrop spectrophotometer (NanoDrop Technologies, Inc., Wilmington, DE).

SNP marker development and genotyping. We used 35 previously reported SNP markers on chromosome 10 (Kang et al. 2014) to localize the $P v r 7$ locus. The linkage between SNP marker and phenotype was analyzed by comparing marker genotype and resistance phenotype using CarthaGene software (Schiex and Gaspin 1997). To develop additional SNP markers, polymerase chain reaction (PCR) primers were designed based on the sequence information of the C. annuum 'CM334' genome sequence (Kim et al. 2014). Amplified PCR products were sequenced by the National Instrumentation Center for Environmental Management (Seoul National University, Korea). Newly identified SNP markers were tested for polymorphism between susceptible and resistant parents, and polymorphic SNP were converted into high-resolution melting (HRM) markers. In total, $916 \mathrm{~F}_{2}$ plants were genotyped by HRM analysis using the Rotor-Gene 6000 Real-Time Rotary Analyzer (Corbett Research, Sydney, Australia).

Fine genetic mapping and identification of candidate genes. Map distances were estimated by determining the frequency of susceptible recombinants among the mapping population. The map was drawn using the MapChart program. SNP markers linked to the $P v r 7$ locus were used to identify a corresponding pepper genome scaffold sequence. Scaffold A was obtained from the pepper genome database (http://passport.pepper.snu.ac.kr/?t=PGD) by BLASTN search against $C$. annuum scaffold version 1.5. Candidate genes were predicted in the target region using the FGENESH tool (Solovyev et al. 2006).

Comparison of Pvr4 and Pvr7. The Pvr4 and $P v r 7$ genes were compared using $P v r 7$ flanking markers and a locus-specific Pvr4 InDel marker (Pvr4-EI-F 5'-CCTGGAAAAGCTATATATC TATTCGGACAT-3' and Pvr4-EI-R 5'-TGGGGGAAGGTAAAA GGAAGCTAA-3'), which was developed in the present study. Marker sequences were PCR amplified from the pepper lines and sequenced by Macrogen (Seoul, Korea Republic). The following pepper lines were screened for polymorphisms: PI159236, CM334, B2110-1A, the commercial heterozygous pepper line IPH10023, and the susceptible cultivar ECW.

Statistical analysis. Statistical analyses were computed and graphs were generated using Microsoft Excel 2013 (Microsoft, Redmond, WA). The $\chi^{2}$ test was performed using GraphPad Prism, version 7.0. $P<0.05$ was considered as statistically significant.

\section{RESULTS}

PepMoV resistance in 9093. PepMoV resistance was tested in 9093, F 1 plants of $9093 \times$ Jeju, and the control PI159236, Jeju, and ECW plants by challenging with PepMoV. The Jeju and ECW susceptible controls uniformly developed bright systemic mosaic symptoms of PepMoV on newly emerged uninoculated and inoculated leaves, whereas no symptoms were observed in the resistant PI159236 and 9093 controls or in $9093 \times$ Jeju $F_{1}$ plants at $10 \mathrm{dpi}$ (Fig. 1A).

Systemic spreading of PepMoV was confirmed in susceptible Jeju and ECW plants by visualizing green fluorescence under UV light, whereas green fluorescence was not detected in 9093, PI159236, or $9093 \times$ Jeju $F_{1}$ plants (Fig. 1A). Systemic infection was further confirmed by ELISA. Viral coat protein accumulated to high levels in inoculated and uninoculated upper leaves of the susceptible Jeju and ECW control plants (Fig. 1B), whereas viral coat protein was not detected in inoculated and uninoculated leaves of 9093 , PI159236, and $9093 \times$ Jeju $F_{1}$ plants (Fig. 1B). These results confirm that PI159236, 9093, and $9093 \times$ Jeju $F_{1}$ plants are resistant to PepMoV (Table 1).

Spectrum of Pvr7-mediated PepMoV resistance in 9093. The $P v r 7$ locus (derived from PI159236) was reported to confer dominant resistance to PepMoV in 9093 (Grube et al. 2000), and PI159236 showed resistance to TEV and TSWV (Dogimont et al. 1996). PepMoV resistance in 9093 was considered to be introgressed from PI159236; therefore, 9093 may display resistance to TEV and TSWV. We performed viral-resistance spectrum analysis to confirm resistance to TEV and TSWV in 9093 (Fig. 2; Table 2).

PI159236 carrying the TSWV resistance gene $T s w$ displayed resistance to TSWV infection (as did PI152225); PI159236 did not 
display any visual symptoms of TSWV infection at 8 dpi. If 9093 was derived from PI159236 (Grube et al. 2000), 9093 also might exhibit resistance to TSWV because $T s w$ and $P v r 7$ loci are tightly linked. Inoculated leaves of either 9093 or $9093 \times$ Jeju $F_{1}$ plants displayed typical symptoms of TSWV infection in uninoculated upper leaves (Fig. 2A). ELISA results confirmed that TSWV coat protein accumulated to high levels in $9093,9093 \times \mathrm{Jeju}_{1}$, and the susceptible Jeju control (Fig. 2B). These results suggest that 9093 likely does not carry the TSWV resistance gene $T s w$. In contrast to the earlier reports of Grube et al. (2000), the present results indicate that 9093 carries only Pvr7.

TEV belongs to the Potyvirus genus. C. chinense 'PI159236' and 'PI152225' express known resistance to TEV. These accessions carry the recessive resistance gene $p v r l$, which is located on chromosome 4. If 9093 was derived from PI159236, 9093 also would be expected to carry $p v r l$. To investigate whether 9093 expresses pvrl, homozygous 9093 and control plants were inoculated with TEV (Fig. 2). The resistant lines PI159236 and PI152225 did not develop viral symptoms in inoculated and upper uninoculated leaves by 7 dpi. By contrast, 9093, $9093 \times$ Jeju F $_{1}$, and Jeju plants displayed clear TEV symptoms in uninoculated upper leaves (Fig. 2A). TEV coat protein did not accumulate in inoculated or uninoculated upper leaves of PI159236 and PI152225. By contrast, TEV coat protein was highly accumulated in both the inoculated and uninoculated upper leaves of 9093 and susceptible control plants (Fig. 2B). These results indicate that 9093 is susceptible to TEV and does not carry pvrl. These combined results confirm that 9093 is susceptible to TSWV and TEV viruses (Table 2), showing virusresistance spectra similar to those of CM334 (Janzac et al. 2009; Moury et al. 2005).

Fine mapping of $\boldsymbol{P v r} 7$. To determine $P v r 7$ inheritance in 9093 , $9169093 \times$ Jeju $F_{2}$ individuals were screened for PepMoV resistance by visualizing GFP fluorescence. ELISA tests were performed to confirm the visual phenotypes. Phenotype analysis of the $9093 \times$ Jeju $F_{2}$ population showed that 688 individuals were resistant and 228 individuals were susceptible to PepMoV, which fit a 3:1 segregation ratio (Table 1). These results confirm that

TABLE 1. Inheritance of Pepper mottle virus (PepMoV) resistance in the $9093 \times$ Jeju $\mathrm{F}_{2}$ population

\begin{tabular}{lrrrrrr}
\hline \multicolumn{7}{c}{$\begin{array}{c}\text { Number of } \\
\text { plants }\end{array}$} \\
\cline { 2 - 4 } Population $^{\mathrm{a}}$ & Total & $\mathrm{R}$ & $\mathrm{S}$ & Expected ratio (R:S) & $\chi^{2}$ & $P$ value \\
\hline Jeju & 10 & 0 & 10 & $0: 1$ & - & - \\
ECW & 10 & 0 & 10 & $0: 1$ & - & - \\
PI159236 & 10 & 10 & 0 & $1: 0$ & - & - \\
9093 & 10 & 10 & 0 & $1: 0$ & - & - \\
$9093 \times$ Jeju $_{1}$ & 5 & 5 & 0 & $1: 0$ & - & - \\
$9093 \times$ Jeju F F $_{2}$ & 916 & 688 & 228 & $3: 1$ & 0.0058 & 0.94 \\
\hline
\end{tabular}

a Parental lines and populations.

b $\mathrm{R}=$ resistant and $\mathrm{S}=$ susceptible.
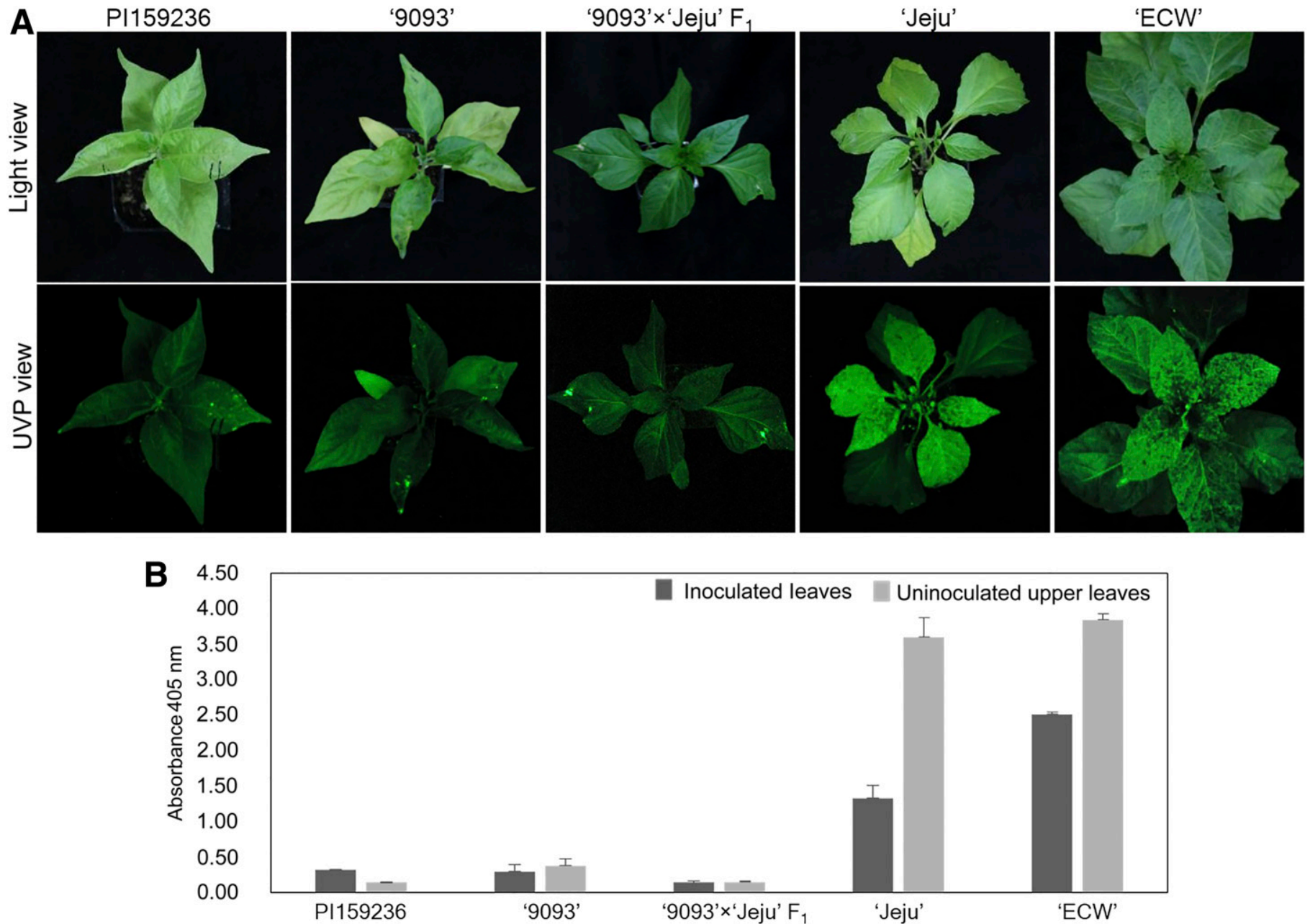

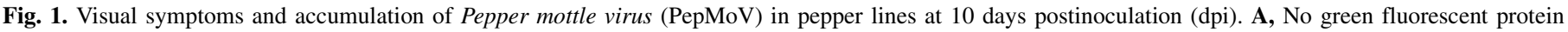

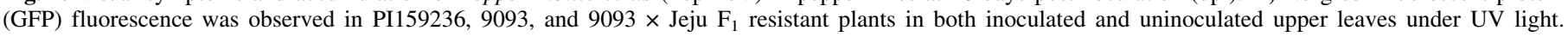

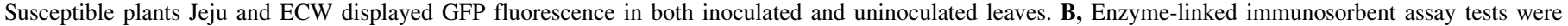
performed at 13 dpi. Data represent an average of three independent experiments. Error bars represent standard deviation of the mean absorbance value. 
PepMoV resistance in 9093 is controlled by a single dominant $P v r 7$ gene (Table 1).

The previous study (Grube et al. 2000) mapped the Pvr7 locus onto chromosome 10 within $4.5 \mathrm{cM}$ on either side of the Pvr4SCAR marker, and showed that $P v r 7$ is less than $2 \mathrm{cM}$ away from Pvr4 (Fig. 3A). In the present study, we used 35 SNP markers that are evenly distributed on pepper chromosome 10 (Kang et al. 2014) to perform fine mapping of the $P v r 7$ locus in the $\mathrm{F}_{2}$ mapping population. In all, 4 of the 35 SNP markers showing clear polymorphism between parental lines and distinguishing PepMoV resistance and susceptibility were converted into the codominant HRM markers. Initial genotyping and segregation analysis revealed that these four SNP markers (SNP-H1.5, SNP-H1.6, SNP-H1.7, and SNP-H1.8) were linked to the Pvr7 locus (Fig. 3B). Additional markers around the $P v r 7$ locus were developed using genomic information from Capsicum spp. For direct sequencing, PCR primers around the $P v r 7$ region in chromosome 10 were designed based on the CM334 genome sequence information (Kim et al. 2014). We also used direct sequencing information to develop four additional HRM SNP markers (SNP-H2.1, SNP-H2.2, SNP-H2.3, and SNP-H2.4), which showed clear polymorphisms (Supplementary Fig. S1). In summary, eight polymorphic codominant markers were used for genotyping the $\mathrm{F}_{2}$ individuals (Table 3 ).

Fine mapping of the $P v r 7$ locus resulted in the identification of 17 recombinant plants: 7, 5, and 3 recombinants for SNP-H2.1, SNPH2.2, and SNP-H2.3, respectively, and 1 recombinant each for SNPH1.7 and SNP-H1.8 (Fig. 3C). These recombinant plants were susceptible to PepMoV. Three of the eight SNP markers (SNP-H2.4, SNP-H1.5, and SNP-H1.6) perfectly cosegregated with the PepMoV-resistant phenotype (Fig. 3C). The Pvr7 locus was

TABLE 2. Summary of resistance response of 9093

\begin{tabular}{lccccc}
\hline & \multicolumn{5}{c}{ Pepper accessions $^{\text {b }}$} \\
\cline { 2 - 6 } Virus $^{\mathrm{a}}$ & $\begin{array}{c}\text { PI159236 } \\
(\mathrm{R}: \mathrm{S})\end{array}$ & $\begin{array}{c}\text { PI152225 } \\
(\mathrm{R}: \mathrm{S})\end{array}$ & $\begin{array}{c}9093 \\
(\mathrm{R}: \mathrm{S})\end{array}$ & $\begin{array}{c}\text { Jeju } \\
(\mathrm{R}: \mathrm{S})\end{array}$ & $\begin{array}{c}9093 \times \text { Jeju } \\
\mathrm{F}_{1}(\mathrm{R}: \mathrm{S})\end{array}$ \\
\hline TSWV & $10: 0$ & $10: 0$ & $0: 10$ & $0: 10$ & $0: 10$ \\
TEV & $10: 0$ & $10: 0$ & $0: 10$ & $0: 10$ & $0: 10$
\end{tabular}

a Tomato spotted wilt virus (TSWV) and Tobacco etch virus (TEV).

b $\mathrm{R}=$ resistant and $\mathrm{S}=$ susceptible plants.
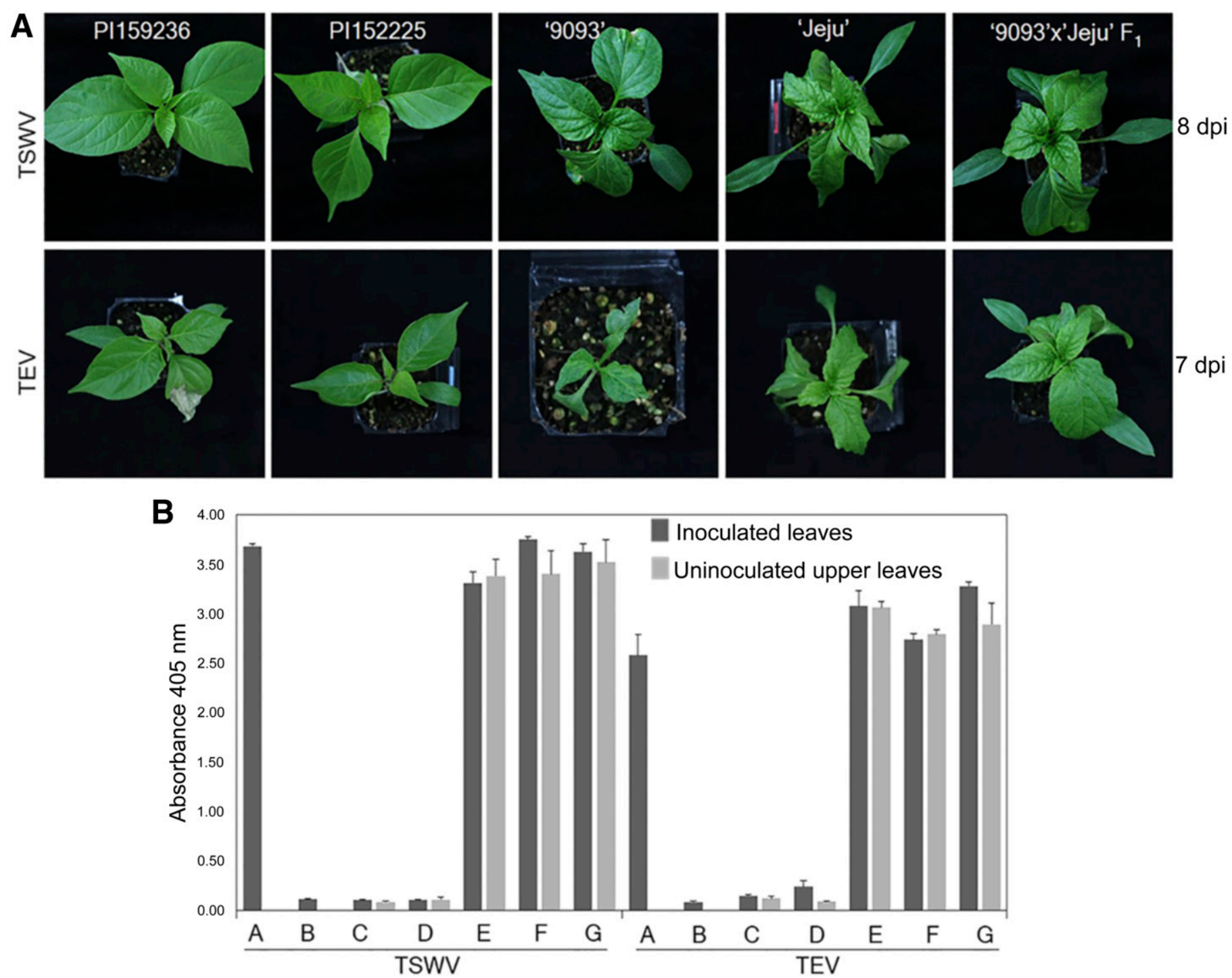

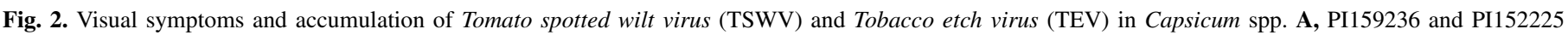

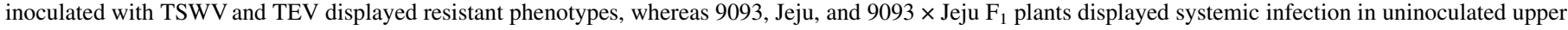

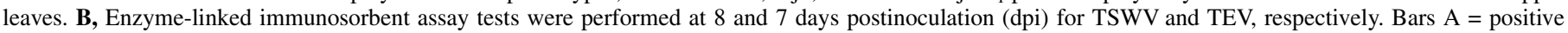

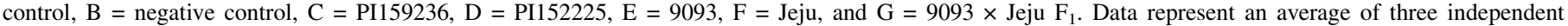
experiments. Error bars represent standard deviation of the mean absorbance value. 
delimited to a genetic interval between the SNP-H2.3 and SNP-H1.7 markers on chromosome 10 (Fig. 3C). The pepper genome sequence information of $C$. annuum 'CM334' (Kim et al. 2014) enabled us to determine that the $P v r 7$ locus was located within the 258-kb region of scaffold A (Fig. 3C). Gene prediction analysis identified nine putative NB-LRR-type $R$ genes within the $P v r 7$ region (Fig. 3C).

Comparison between Pvr4 and Pvr7. Molecular mapping analysis localized the $P v r 7$ locus in a genomic region where the Pvr4 locus was recently mapped in the CM334 genome (Kim et al. 2017). This prompted us to investigate the genetic relationship between $P v r 4$ - and $P v r 7$-mediated PepMoV resistance. Therefore, we genotyped pepper plants using the SNP markers flanking the Pvr7 locus (SNP-H2.3 and SNP-H1.7). Sequence analysis of the SNP-H2.3 marker showed no polymorphism among the pepper lines CM334, 9093, IPH10023, and B2110-1A (source of Pvr7). By contrast, SNP-H1.7 was polymorphic, and genotypes of 9093 and
B2110-1A were found to be the same as that of CM334 (Table 4), suggesting that $P v r 7$ could be derived from CM334.

The specific InDel marker developed for the Pvr4 locus in the present study was used for genotyping pepper lines (Supplementary Fig. S2). Pvr4-specific InDel marker amplification was observed in 9093, B2110-1A, and the commercial $F_{1}$ hybrid (IPH10023), which are known to carry the $P v r 7$ locus. However, no amplification was observed in ECW or PI159236, which was reported as the source of $P v r 7$ (Grube et al. 2000). These results further confirm that PI159236 is not a source of socalled Pvr7-mediated PepMoV resistance. The actual source of resistance to PepMoV in 9093 could be introgressed from C. annuum, probably via CM334, which is known to carry Pvr4, a dominant resistance gene (Kim et al. 2017; Kyle and Palloix 1997). Therefore, these combined results suggest that Pvr4 and Pvr7 should be considered as the same locus derived from $C$. апnиит 'CM334'.

A
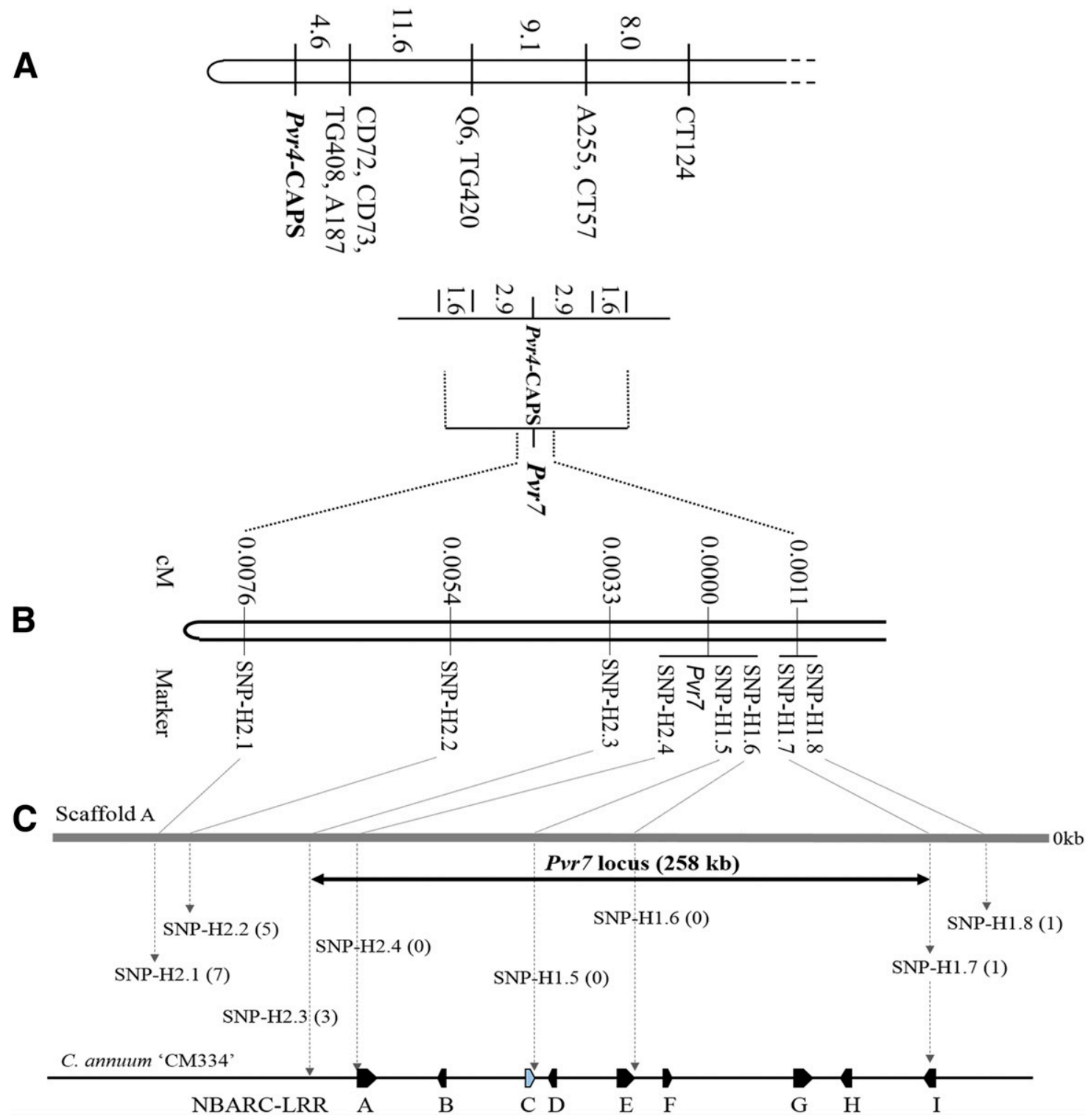

Fig. 3. Fine mapping of the potyvirus resistance gene $P v r 7$. A, Linkage map of the $P v r 7$ locus. B, Linkage map of the $P v r 7$ locus that was developed based on the $9093 \times$ Jeju $F_{2}$ population in the present study. Numbers above the chromosome indicate genetic distances in centimorgans. Single-nucleotide polymorphism (SNP) markers linked to the $P v r 7$ locus are indicated below the chromosome. C, Fine mapping of the $P v r 7$ locus. The $P v r 7$ locus was delimited to a 258 -kb region between the SNP-H2.3 and SNP-H1.7 markers on the Capsicum annuum 'CM334' scaffold A. Numbers in parentheses indicate the numbers of recombinant plants identified. A to I indicate the predicted nucleotide-binding leucine-rich repeat (NB-LRR) candidate genes. The candidate gene corresponding to the Pvr4 gene is indicated in the light-shaded box. 


\section{DISCUSSION}

Two dominant potyvirus $R$ genes, $P v r 4$ and $P v r 7$, are reported to be tightly linked. They are believed to have originated from different sources, with Pvr4 originally reported to confer PVY and PepMoV resistance from C. annuum 'CM334' and Pvr7 reported for resistance to PepMoV from C. chinense 'PI159236' (Grube et al. 2000; Kyle and Palloix 1997). The $P v r 7$ locus was mapped to a genetic interval of $9 \mathrm{cM}$ on chromosome 10 (Grube et al. 2000). A previous study reported that $P v r 4$ and $P v r 7$ have the same inheritance mechanism but are not allelic due to the recovery of a small number of fully susceptible plants in an $\mathrm{R} \times \mathrm{R}$ segregating population (Grube et al. 2000). The present study aimed to perform fine mapping of the $P v r 7$ locus and elucidate the precise genetic relationship between $P v r 4$ and $P v r 7$.

Fine mapping of the $P v r 7$ locus using a segregating population of $916 \mathrm{~F}_{2}$ plants from the $9093 \times$ Jeju cross delimited the $P v r 7$ locus to a 258-kb region between two flanking SNP markers (SNP-H2.3 and SNP-H1.7) on chromosome 10. This localized the $P v r 7$ locus in the same genomic interval that was previously mapped for the Pvr4 locus in the CM334 genome (Kim et al. 2017). The Pvr4 mapping results of Kim et al. (2017) also point to a cluster of multiple NBLRR genes (NBARC-LRR A to I) that were predicted from the Pvr7 target region.

Pvr7-mediated PepMoV resistance in 9093 was reported to be derived from a repeated backcross of $C$. chinense PI159236 with an unknown recurrent bell pepper parent (C. annuum) (Grube et al. 2000), and the donor of the PepMoV resistance was considered to be C. chinense PI159236. However, the fine-mapping results for Pvr7 and the viral-resistance spectrum of 9093 clearly suggest that PepMoV resistance in 9093 might be derived from an unknown parental line rather than $C$. chinense 'PI159236'. If 9093 is derived from PI159236, then TSWV resistance also would be expected in 9093 because PI159236 carries the Tsw locus (Grube et al. 2000; Kim et al. 2017), which is very tightly linked to $P v r 7$. However, 9093 and $9093 \times$ Jeju F 1 plants were susceptible to TSWV. Previous genetic analyses of PepMoV resistance in pepper varieties (Murphy et al. 1998; Subramanya 1982) have also demonstrated the recessive

TABLE 3. Markers used for fine mapping of the $P v r 7$ locus

\begin{tabular}{ll}
\hline Marker $^{\mathrm{a}}$ & \multicolumn{1}{c}{ Sequence } \\
\hline SNP-H2.1-F & GCTTTCTCAACTCTGCCACAA \\
SNP-H2.1-R & TCTCATTCGAACGTGAATTTGTT \\
SNP-H2.2-F & AGCCCATGAGGAATGAGGTG \\
SNP-H2.2-R & CTGGGTCCGCCTTGTATGAT \\
SNP-H2.3-F & GGTCCGCTGATCAATTTTGG \\
SNP-H2.3-R & GTTGTCCCAAGGAGGTTGGT \\
SNP-H1.7-F & TTGGCCCAAAGTCCATTTCTT \\
SNP-H1.7-R & TCAGTGGAACACGTCAGGCA \\
SNP-H1.8-F & GCATGTACTTTGATTAAATTCCACGG \\
SNP-H1.8-R & CAAGCTAACCCAAAGTAAATAAAGCAA \\
\hline
\end{tabular}

${ }^{a}$ Single-nucleotide polymorphism (SNP) markers, SNP-H2.4, SNP-H1.5, and SNP-H1.6 correspond to the SNP markers 575, 61786, and 17918 from Kim et al. (2017).

TABLE 4. Sequence analysis of the single-nucleotide polymorphism (SNP) markers, SNP-H1.7 and SNP-H2.3

\begin{tabular}{llllllc}
\hline & \multicolumn{5}{c}{ SNP-H1.7 } & \\
\cline { 2 - 5 } Plant & 1 & 2 & 3 & 4 & 5 & SNP-H2.3 \\
\hline IPH10023 (Hetero) & G/T & C/T & T/C & C/T & G/A & T \\
B2110-1A (Homo) & G & C & T & C & G & T \\
CM334 & G & C & T & C & G & T \\
9093 & G & C & T & C & G & T \\
\hline
\end{tabular}

a SNP positions within the high-resolution melting marker are indicated from 1 to 5 . resistance of PI159236 against PepMoV. Thus, all these studies indicate the mischaracterization of the dominant PepMoV resistance in 9093.

If, as we conclude, the PepMoV $R$ genes $P v r 4$ and $P v r 7$ from CM334 and 9093 are the same gene, then no susceptible individuals would be expected in the test-cross populations derived from a cross between $9093 \times \mathrm{CM} 334 \mathrm{~F}_{1}$ and Jupiter or RNaky (Grube et al. 2000). Genetic analyses of these test-cross populations recovered five and seven susceptible plants among 826 RNaky- and 905 Jupiter-derived test-cross plants, with recombination frequencies of 0.0121 and 0.0155, respectively (Grube et al. 2000). Therefore, Grube et al. (2000) proposed that PepMoV resistance from CM334 and 9093 is conditioned by two different genes, Pvr4 and Pvr7, linked at less than $2 \mathrm{cM}$. However, it was not conclusive whether $P v r 7$ and $P v r 4$ are allelic or the same locus because the linkage between $P v r 7$ and $P v r 4$ loci has not been directly confirmed. Those unexpected recombinants in test-cross populations may be caused by unequal crossing-over following paring between duplicated sequences, similar to the case in the maize complex resistance gene locus $R p 1$, which consists of multiple genes (Hulbert and Bennetzen 1991; Hulbert et al. 1993), or simply breakdown of resistance in otherwise genetically resistant individuals in the disease screen. It was suggested that unequal exchange events would result in the mapping of genes to different relative positions even if the genes were identical or allelic (Hulbert et al. 1993), and could lead to misidentification of the alleles or genes. To avoid such problems, the sequences of the flanking markers or the gene itself should be verified in addition to performing genetic linkage analysis to unravel the genetic relationship between closely linked genes or multiple allelic variants.

To reveal the genetic relationship between $P v r 4$ and $P v r 7$, we compared $P v r 7$ flanking marker sequences with the $P v r 4$ gene using pepper lines that were known sources of $P v r 7$ and $P v r 4$. First, if 9093 carried the $P v r 7$-introgressed region from PI159236, no $P v r 4$ specific InDel marker amplification would be expected. Second, the genotype of $P v r 7$ flanking markers should be similar to that of C. chinense 'PI159236'. However, Pvr4-specific InDel marker amplification was observed only in 9093, B2110-1A, and the commercial $\mathrm{F}_{1}$ hybrid (IPH10023) that carries the $P v r 7$ locus. The genotype of the SNP-H1.7 flanking marker was similar to that of CM334. These results combined with $P v r 7$ fine mapping and viralresistance spectrum analysis of 9093 clearly demonstrate that the actual source of PepMoV resistance in 9093 could be derived from CM334, which is known to carry the dominant $P v r 4$ resistance gene (Kim et al. 2017; Kyle and Palloix 1997).

Analysis of genetic relationships between potyvirus $R$ genes and their resistance spectrum has been complicated because of the use of unrelated or uncharacterized resistance sources and the existence of complex loci. In the present study, viral-resistance spectrum analysis of 9093, which carries $P v r 7$, revealed resistance responses similar to those of CM334 against known pepper viruses. The $P v r 7$ locus was fine mapped in the same genomic interval of chromosome 10 in CM334 in which Pvr4 was localized. Genotyping of flanking markers and gene-specific InDel markers of $P v r 4$ or $P v r 7$ revealed identical genotypes for 9093 and CM334. Thus, this work confirmed that $P v r 4$ and $P v r 7$ are the same gene, with both originating from CM334. These combined results demonstrate the importance and utility of the flanking markers and gene-specific markers in the genetic dissection of complex loci.

\section{ACKNOWLEDGMENTS}

This research was supported by Golden Seed Project (213006-05-1SB540), Ministry of Agriculture, Food and Rural Affairs, Ministry of Oceans and Fisheries, Rural Development Administration, and Korea Forest Service, Republic of Korea and by "Cooperative Research Program for Agriculture Science \& Technology Development (Project Number PJ01120401)" Rural Development Administration, Republic of Korea. 


\section{LITERATURE CITED}

Abdalla, O., Desjardins, P., and Dodds, J. 1991. Identification, disease incidence, and distribution of viruses infecting peppers in California. Plant Dis. 75:1019-1023.

Caranta, C., Palloix, A., Gebre-Selassie, K., Lefebvre, V., Moury, B., and Daubeze, A. M. 1996. A complementation of two genes originating from susceptible Capsicum апnиит lines confers a new and complete resistance to pepper veinal mottle virus. Phytopathology 86:739-743.

Caranta, C., Thabuis, A., and Palloix, A. 1999. Development of a CAPS marker for the Pvr4 locus: A tool for pyramiding potyvirus resistance genes in pepper. Genome 42:1111-1116.

Dogimont, C., Palloix, A., Daubze, A. M., Marchoux, G., Selassie, K. G., and Pochard, E. 1996. Genetic analysis of broad spectrum resistance to potyviruses using doubled haploid lines of pepper (Capsicum anпиит L.). Euphytica 88:231-239.

Grube, R. C., Blauth, J. R., Andrés, M. A., Caranta, C., and Jahn, M. K. 2000. Identification and comparative mapping of a dominant potyvirus resistance gene cluster in Capsicum. Theor. Appl. Genet. 101:852-859.

Guerini, M. N., and Murphy, J. F. 1999. Resistance of Capsicum annuum 'Avelar' to pepper mottle potyvirus and alleviation of this resistance by co-infection with cucumber mosaic cucumovirus are associated with virus movement. J. Gen. Virol. 80:2785-2792.

Hulbert, S. H., and Bennetzen, J. L. 1991. Recombination at the Rpl locus of maize. Mol. Gen. Genet. 226:377-382.

Hulbert, S. H., Sudupak, M. A., and Hong, K. S. 1993. Genetic relationships between alleles of the Rpl rust resistance locus of maize. Mol. PlantMicrobe Interact. 6:387-392.

Hwang, J., Li, J., Liu, W. Y., An, S. J., Cho, H., Her, N. H., Yeam, I., Kim, D., and Kang, B. C. 2009. Double mutations in eIF4E and eIFiso4E confer recessive resistance to Chilli veinal mottle virus in pepper. Mol. Cells 27:329-336.

Jahn, M., Paran, I., Hoffmann, K., Radwanski, E. R., Livingstone, K. D., Grube, R. C., Aftergoot, E., Lapidot, M., and Moyer, J. 2000. Genetic mapping of the Tsw locus for resistance to tomato spotted wilt virus in Capsicum and its relationship to the $S w-5$ gene for resistance to the same pathogen in tomato. Mol. Plant-Microbe Interact. 13:673-682.

Janzac, B., Fabre, M. F., Palloix, A., and Moury, B. 2009. Phenotype and spectrum of action of the Pvr4 resistance in pepper against potyviruses, and selection for virulent variants. Plant Pathol. 58:443-449.

Kang, B. C., Yeam, I., Frantz, J. D., Murphy, J. F., and Jahn, M. M. 2005. The pvrl locus in Capsicum encodes a translation initiation factor eIF4E that interacts with Tobacco etch virus VPg. Plant J. 42:392-405.

Kang, J. H., Yang, H. B., Jeong, H. S., Choe, P., Kwon, J. K., and Kang, B. C. 2014. Single nucleotide polymorphism marker discovery from transcriptome sequencing for marker-assisted backcrossing in Capsicum. Korean J. Hortic. Sci. Technol. 32:535-543.

Kang, W. H., Hoang, N. H., Yang, H. B., Kwon, J. K., Jo, S. H., Seo, J. K., Kim, K. H., Choi, D., and Kang, B. C. 2010. Molecular mapping and characterization of a single dominant gene controlling CMV resistance in peppers (Capsicum annuum L.). Theor. Appl. Genet. 120:1587-1596.

Kim, H. J., Han, J. H., Kim, S., Lee, H. R., Shin, J. S., Kim, J. H., Cho, J., Kim, Y., Lee, H., Kim, B., and Choi, D. 2011. Trichome density of main stem is tightly linked to PepMoV resistance in chili pepper (Capsicum annuиm L.). Theor. Appl. Genet. 122:1051-1058.

Kim, M. S., Kim, M. J., Hong, J. S., Choi, J. K., and Ryu, K. H. 2010. Patterns in disease progress and the influence of single and multiple viral infections on pepper (Capsicum annuum L.) growth. Eur. J. Plant Pathol. 127:53-61.

Kim, S., Park, M., Yeom, S.-I., Kim, Y.-M., Lee, J. M., Lee, H.-A., Seo, E., Choi, J., Cheong, K., Kim, K.-T., Jung, K., Lee, G.-W., Oh, S.-K., Bae, C., Kim, S.-B., Lee, H.-Y., Kim, S.-Y., Kim, M.-S., Kang, B.-C., Jo, Y. D., Yang, H.-B., Jeong, H.-J., Kang, W.-H., Kwon, J.-K., Shin, C., Lim, J. Y., Park, J. H., Huh, J. H., Kim, J.-S., Kim, B.-D., Cohen, O., Paran, I., Suh, M. C., Lee, S. B., Kim, Y.-K., Shin, Y., Noh, S.-J., Park, J., Seo, Y. S., Kwon, S.-Y., Kim, H. A., Park, J. M., Kim, H.-J., Choi, S.-B., Bosland, P. W., Reeves, G., Jo, S.-H., Lee, B.-W., Cho, H.-T., Choi, H.-S., Lee, M.-S., Yu, Y., Choi, Y. D., Park, B.-S., van Deynze, A., Ashrafi, H., Hill, T., Kim, W. T., Pai, H.-S., Ahn, H. K., Yeam, I., Giovannoni, J. J., Rose, J. K. C.,
Sørensen, I., Lee, S.-J., Kim, R. W., Choi, I.-Y., Choi, B.-S., Lim, J.-S., Lee, Y.-H., and Choi, D. 2014. Genome sequence of the hot pepper provides insights into the evolution of pungency in Capsicum species. Nat. Genet. 46:270-278.

Kim, S.-B., Kang, W.-H., Huy, H. N., Yeom, S.-I., An, J.-T., Kim, S., Kang, M.-Y., Kim, H. J., Jo, Y. D., Ha, Y., Choi, D., and Kang, B.-C. 2017. Divergent evolution of multiple virus-resistance genes from a progenitor in Capsicum spp. New Phytol. 213:886-899.

Kim, Y. J., Jonson, M. G., Choi, H. S., Ko, S. J., and Kim, K. H. 2009. Molecular characterization of Korean Pepper mottle virus isolates and its relationship to symptom variations. Virus Res. 144:83-88.

Kyle, M. M., and Palloix, A. 1997. Proposed revision of nomenclature for potyvirus resistance genes in Capsicum. Euphytica 97:183-188.

Lamb, E. M., Atkins, S., Schuler, K. D., and Roberts, P. D. 2001. Pepper mild mottle virus. University of Florida, IFAS Ext. Bull. 808.

Lee, H. R., An, H. J., You, Y. G., Lee, J., Kim, H. J., Kang, B. C., and Harn, C. H. 2013. Development of a novel codominant molecular marker for Chili veinal mottle virus resistance in Capsicum annuum L. Euphytica 193: 197-205.

Moury, B., Palloix, A., Caranta, C., Gognalons, P., Souche, S., Selassie, K. G., and Marchoux, G. 2005. Serological, molecular, and pathotype diversity of Pepper veinal mottle virus and Chili veinal mottle virus. Phytopathology 95:227-232.

Murphy, J. F., Blauth, J. R., Livingstone, K. D., Lackney, V. K., and Jahn, M. K. 1998. Genetic mapping of the pvrl locus in Capsicum spp. and evidence that distinct potyvirus resistance loci control responses that differ at the whole plant and cellular levels. Mol. Plant-Microbe Interact. 11: 943-951.

Murphy, J. F., and Kyle, M. M. 1995. Alleviation of restricted systemic spread of pepper mottle potyvirus in Capsicum annuum cv. Avelar by coinfection with a cucumovirus. Phytopathology 85:561-566.

Parrella, G., Ruffel, S., Moretti, A., Morel, C., Palloix, A., and Caranta, C. 2002. Recessive resistance genes against potyviruses are localized in colinear genomic regions of the tomato (Lycopersicon spp.) and pepper (Capsicum spp.) genomes. Theor. Appl. Genet. 105:855-861.

Rialch, N., Sharma, V., Sharma, A., and Sharma, P. N. 2015. Characterization and complete nucleotide sequencing of Pepper mild mottle virus infecting bell pepper in India. Phytoparasitica 43:327-337.

Rodríguez-Alvarado, G., Fernandez-Pavia, S., Creamer, R., and Liddell, C. 2002. Pepper mottle virus causing disease in chile peppers in southern New Mexico. Plant Dis. 86:603-605.

Ruffel, S., Dussault, M. H., Palloix, A., Moury, B., Bendahmane, A., Robaglia, C., and Caranta, C. 2002. A natural recessive resistance gene against potato virus $\mathrm{Y}$ in pepper corresponds to the eukaryotic initiation factor 4E (eIF4E). Plant J. 32:1067-1075.

Ruffel, S., Gallois, J. L., Moury, B., Robaglia, C., Palloix, A., and Caranta, C. 2006. Simultaneous mutations in translation initiation factors eIF4E and eIF(iso)4E are required to prevent pepper veinal mottle virus infection of pepper. J. Gen. Virol. 87:2089-2098.

Schiex, T., and Gaspin, C. 1997. Carthagene: Constructing and joining maximum likelihood genetic maps. Proc 5th Int. Conf. ISMB.

Solovyev, V., Kosarev, P., Seledsov, I., and Vorobyev, D. 2006. Automatic annotation of eukaryotic genes, pseudogenes and promoters. Genome Biol. $7: 10.1-10.12$.

Subramanya, R. 1982. Relationship between tolerance and resistance to pepper mottle virus in a cross between Capsicum annuum L. $\times$ Capsicum chinense Jacq. Euphytica 31:461-464.

Valkonen, J. P. T., Kyle, M. M., and Slack, S. A. 1996. Comparison of resistance to potyviruses within Solanaceae: Infection of potatoes with tobacco etch potyvirus and peppers with potato A and Y potyviruses. Ann. Appl. Biol. 129:25-38.

Warren, C. E., and Murphy, J. F. 2003. The complete nucleotide sequence of Pepper mottle virus-Florida RNA. Arch. Virol. 148:189-197.

Yeam, I., Kang, B. C., Lindeman, W., Frantz, J. D., Faber, N., and Jahn, M. M. 2005. Allele-specific CAPS markers based on point mutations in resistance alleles at the pvrl locus encoding eIF4E in Capsicum. Theor. Appl. Genet. 112:178-186. 